\title{
Scaling of Superconducting Switches for Extraction of Magnetic Energy
}

\author{
Amalia Ballarino and Thomas Taylor
}

\begin{abstract}
In certain cases it is necessary to extract the energy from a superconducting magnet when it quenches, in order to limit the heat generated by the event and thus prevent irreversible damage. This is usually achieved by opening a contact breaker across a resistor in the circuit feeding the magnet. For the heavy currents used to excite large magnets such switches incorporate sophisticated devices to limit arcing during the operation; the devices are reliable but are also bulky and expensive. It is interesting to consider the use of superconducting switches to perform this function, an important advantage being that they would be housed in the cryogenic environment of the magnet, and thus avoid permanent diversion of the current in and out of that environment to the mechanical switch (which operates at room temperature). However, practical switches for such an application are made up of superconductor in a metal matrix, and it is convenient to work with a relatively low resistance to approximate to the open circuit. This leads to scaling laws for superconducting switches for this application which relate the operating current and stored energy of the magnet system, the type of superconductor, and the necessary size of the device.
\end{abstract}

Index Terms-Energy extraction, magnet protection, superconducting switch.

\section{INTRODUCTION}

$\mathbf{T}$ HE usual way of extracting the energy from large superconducting (SC) magnet systems is to divert the current through power leads to room-temperature dump resistors having circuit breakers in parallel. During normal operation the current passes through the closed circuit breaker, which implies that if several resistors are required (to limit the maximum voltage), the system has to be fitted with extra power leads that pass current continuously. It is therefore interesting to consider the possibility of putting the switch into the cryogenic environment (see Fig. 1). If the switch is superconducting there is no power dissipated during normal running, and the safety leads required to connect the switch to the room temperature dump resistor only need to conduct current during the discharge of the energy: they can therefore be designed to conduct less heat into the magnet system than leads that have to transfer current continuously.

Switches for this application would need to be very reliable, and it is assumed that they would be made from superconductor in a metal matrix or on a metallic substrate. However, both the resistive alloys chosen for this purpose and the superconducting

Manuscript received October 13, 2009. First published April 05, 2010; current version published May 28, 2010

The authors are with CERN, European Organization for Nuclear Research, 1211 Geneva 23, Switzerland (e-mail: Amalia.ballarino@cern.ch; Thomas.taylor@cern.ch).

Digital Object Identifier 10.1109/TASC.2010.2042044

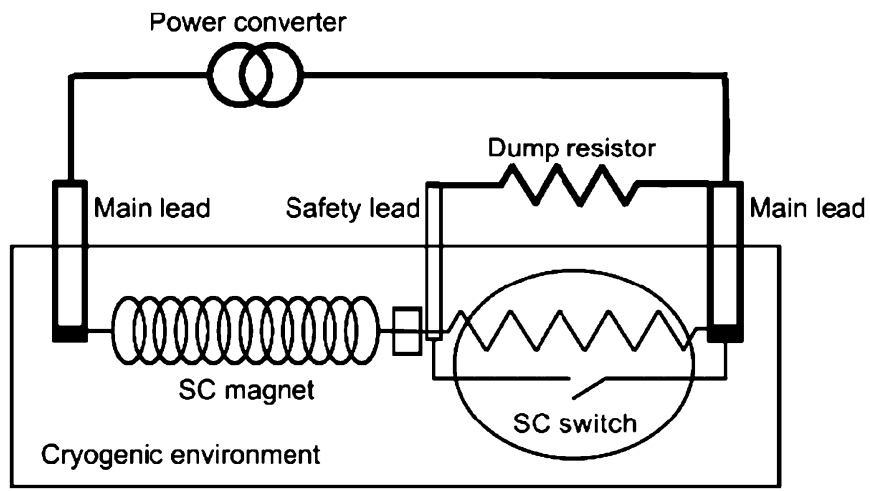

Fig. 1. Basic circuit using a superconducting switch to divert a large fraction of the discharged energy to a dump resistor at room temperature.

material (driven normal) will carry some fraction of the current in the "off" state. Since the cross-section of the switch conductor is determined by the maximum current it is required to carry in the steady state, an increase in the resistance of the switch for a given circuit will require a longer length, and therefore a greater mass of material. The minimum mass required is determined by the maximum fraction of the total energy to be extracted from the system being protected that can be safely absorbed by the switch. It is therefore possible to calculate the minimum size of the switches that would be required, as a function of stored energy, operating current and discharge voltage, while taking into account the characteristics of the conductor materials used in the device.

As circuit breakers and power leads are reliable classical devices, it would clearly be necessary to evaluate carefully the cost and benefit of embarking on the necessary program to develop appropriate superconducting switches. The purpose of this report is to provide guidelines for sizing the switches and comparing the different materials, as input for deciding whether or not to proceed with such $R \& D$.

\section{Assumptions}

General assumptions concerning the switch considered here correspond to those that would apply, for example, to a superconducting magnet system associated with a particle accelerator, and can be summarized as follows:

- The cross-section, $A$, of the switch conductor carries safely the maximum current in the main circuit, $I_{0}$

- The switch conductor is quenched using direct heat or overcurrent. The energy, $E_{a}$, required to switch is less than the energy, $E_{0}$, stored in the main circuit

- The switch consists of a non-inductive winding that withstands repeated warming to temperature $T_{\max }$ 
- The circuit is discharged into the external resistor, $R_{e}$, in parallel with the open switch in the resistive state, resistance $R_{s}$, at maximum specified voltage $V_{0}$

- The total length of the switch conductor is $S$

- The switch heats up adiabatically during a period corresponding to the time constant of the circuit

- After opening, the switch is latched "off" by heating due to the main current.

The switch is dimensioned such that its temperature does not exceed $T_{\max }$ when the stored energy is discharged into the combined resistance, $R$, of the circuit. Initially $V_{0}=I_{0} R$, and then voltage and current decay exponentially with time constant $\tau=L / R=L I_{0} / V_{0}$, where $L$ is the inductance.

The resistance of the switch $R_{s}=\rho(T) S / A$, where $\rho(T)$ is the effective resistivity of the combination of materials in the switch conductor, and the current flowing through it at time $t$ after firing is $I_{0}\left(R_{e} /\left(R_{s}+R_{e}\right)\right) e^{-t / \tau}$. The energy that must be absorbed by the switch, $E_{s}=$ $\int R_{s}\left(I_{0}\left(R_{e} /\left(R_{s}+R_{e}\right)\right)\right)^{2} e^{-2 t / \tau} d t$. For materials used to make switches, such as $\mathrm{Cu}-\mathrm{Ni}$, resistivity is weakly dependent on temperature, so it can be assumed that $\rho$, and consequently $R_{s}$, remain constant. Integrating from $t=0$ to $\infty$ and putting $R=R_{s} R_{e} /\left(R_{s}+R_{e}\right)$, gives

$$
E_{s}=\frac{L R I_{0}^{2}}{2^{R_{s}}} .
$$

The energy that can be safely absorbed by the switch cable is determined by its mass, $M_{s}$, and the integral of the specific heat from $T_{0}$ to $T_{\max }$, where $T_{0}$ is the initial temperature (i.e. when the switch opens). Calling this quantity $C_{T}$, the energy that can be absorbed is $M_{s} C_{T}$, and this must be greater than $E_{s}$.

The cross-section of the switch cable is dimensioned so as to carry the full current safely in the superconducting mode. If $J_{\text {eng }}$ is the engineering current density limit in the switch, then

$$
A=\frac{\sigma I_{0}}{J_{\text {eng }}},
$$

where $\sigma$ is a safety factor to ensure sufficient margin.

Substituting $V_{0} / I_{0}$ for $R$ in (1), $M_{s} C_{T}$ for $E_{s}$, and putting $M_{s}=\nu S A$ gives $R_{s} \geq L I_{0} V_{0} /(2 v A S C T)$. The parameter $R_{s}$ can then be eliminated to give convenient expressions for the length, $S$, of conductor required,

$$
S \geq \sqrt{\frac{L V_{0} I_{0}}{2 v \rho C_{T}}},
$$

and total mass of wire or cable in the switch,

$$
M_{s} \geq \sqrt{\frac{E_{0} I_{0} V_{0} v \sigma^{2}}{\left(J_{\text {eng }}^{2} \rho C_{T}\right)}},
$$

in terms of parameters of the magnetic circuit and switch. The other parameters can be derived using (1)to (3).

\section{EXAMPLES AND SCALING}

\section{A. A Typical Case}

In applications for accelerators, the most likely candidates for using a superconducting switch for energy extraction are magnetic circuits having stored energy in the range $100 \mathrm{~kJ}$ to 100 MJ. As an example, we consider a magnet with a stored energy of $2 \mathrm{MJ}$, running at a maximum current of $2 \mathrm{kA}$, and built to withstand discharging at $1 \mathrm{kV}$. For the switch the initial choice would be classical multi-filamentary $\mathrm{Nb}-\mathrm{Ti}$ in a $\mathrm{Cu}-30 \mathrm{Ni}$ matrix, with a ratio of matrix to superconductor of $1: 1$. The resistivity of the matrix material is $35 \times 10^{-8} \Omega \cdot \mathrm{m}$, almost constant between $4 \mathrm{~K}$ and $250 \mathrm{~K}$; and that of Nb-Ti is $60 \times 10^{-8} \Omega \cdot \mathrm{m}$ at $10 \mathrm{~K}$, increasing to $65 \times 10^{-8} \Omega \cdot \mathrm{m}$ at $150 \mathrm{~K}$. The maximum field on any conductor in the non-inductively wound switch coil can be less than $3 \mathrm{~T}$, so it is reasonable to assume $J_{\text {eng }}=1.5 \times 10^{9} \mathrm{~A} / \mathrm{m}^{2}$ when the switch is cooled by liquid helium at $4.5 \mathrm{~K}$. For this conductor the margin must be large [1], say $\sigma=3$. The average density, $v=7.5 \times 10^{3} \mathrm{~kg} / \mathrm{m}^{3}$. In going from $10 \mathrm{~K}$ to $150 \mathrm{~K}$, $C_{T}=2 \times 10^{4} \mathrm{~J} / \mathrm{kg}$; by increasing the maximum temperature to $250 \mathrm{~K}$ this value is doubled. For this example, a superconducting switch having maximum safe working temperature of $150 \mathrm{~K}$ would require a conductor mass of about $3.4 \mathrm{~kg}$, and if the switch could be made for safe use up to $250 \mathrm{~K}$ the mass could be reduced to about $1.7 \mathrm{~kg}$.

\section{B. Scaling}

For scaling purposes it is convenient to split the expression for the total mass of the switch into two components, one pertaining solely to the magnetic circuit, $f_{1}=\sqrt{ }\left(E_{0} I_{0} V_{0}\right)$, and the other, $f_{2}=\left(\sigma / J_{\text {eng }}\right) \sqrt{ }\left(\nu / \rho C_{T}\right)$, pertaining solely to the characteristics of the switch: then the minimum of $M_{s}=f_{1} f_{2}$. For example, using the same switch technology as that taken for the example above, only $f_{1}$ is affected and it can be readily shown that a switch for a circuit operating at $15 \mathrm{kA}$ and having a stored energy of $100 \mathrm{MJ}$ would need 20 times as much superconducting material, i.e. of the order of $34 \mathrm{~kg}$ for a switch designed to operate safely and repeatedly up to $250 \mathrm{~K}$. When comparing switches based on the use of different conductors, it is sufficient to concentrate on comparing $f_{2}$.

\section{Switch TRANSITION}

Triggering the switch is accomplished by rapidly moving the working point of the conductor outside the critical surface, by raising the temperature, current density, or magnetic field. Use of a pulsed magnetic field for this purpose can be excluded as being impractical for large switches. Heat pulses via quench heaters are widely used for protecting magnets, and this technique is clearly a candidate: the problem is that for rapid effect the heater must be separated from the winding by a thin layer of insulation, and as the quench propagation velocities in the low conductivity alloy matrices are low it has to be co-wound to provide a large area of contact. This method has been demonstrated to work for small HTS switches [2], and substantial experience with quench heaters for magnet protection ensures mastery of the technology. However, for large and robust switches insulation could be problematic, both with regard to breakdown and the energy required to raise the temperature sufficiently quickly. 


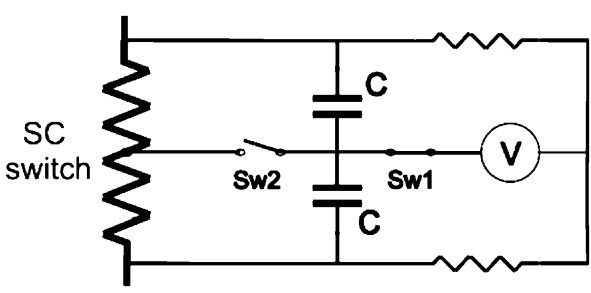

Fig. 2. Schematic for quenching the switch by over-current. Capacitors $\mathrm{C}$ are kept charged via $\mathrm{Sw} 1$. When it is required to drive the superconducting switch (on the left) normal, Sw1 is opened and Sw2 is closed, and the capacitors are discharged into the winding.

Other methods of providing heat have been suggested, e.g. radiation [3]. But the most attractive way to drive switches of this type normal is probably to use a large current pulse [4]. This is achieved by discharging capacitors via a center tap of the (non-inductive) switch as illustrated in Fig. 2. The method is efficient, as all the energy is deposited where it is required - directly into the conductor. In addition, the turn-to-turn insulation of the switch can be optimized without being compromised by installing heaters everywhere. However, in order to extract energy when the magnet is operating at low current it may be necessary to include some heating to latch the switch in the off state.

\section{USE OF MATERIALS OTHER THAN NiOBIUM-TITANIUM}

Superconducting switches were widely studied in the 1970s and 1980s, and it was then that multi-filamentary Nb-Ti in a cupronickel matrix was chosen. While this material can be readily cabled or braided with full transposition, and is easy to handle mechanically and relatively inexpensive, it has low minimum quench energy. This is due to the high resistivity and low heat capacity of the matrix at the working temperature of around $5 \mathrm{~K}$, and means that the switch has to be very carefully assembled to avoid small movements. While this may be good for switching, in order to avoid spurious quenching the maximum operating current has to be chosen to provide a substantial temperature margin [1]. With their higher operating temperatures the minimum quench energy of HTS materials is much larger, so despite their having less favorable mechanical properties, it is worth considering their application to switches [5]. With this in mind, a recent study [2] has addressed of the application of HTS to switches, with particular regard to their use to discharge magnets having stored energy of up to some tens of kJ. In such cases the switch itself can be used to absorb all the energy. For the much larger systems discussed here an external dump resistor is mandatory, and in this section the use of HTS for making the switches is compared with that of standard $\mathrm{Nb}-\mathrm{Ti}$.

\section{A. Magnesium-Diboride}

The critical temperature of $\mathrm{MgB}_{2}$ is $39 \mathrm{~K}$. As specific heat in the temperature range up to $40 \mathrm{~K}$ varies as the cube of the temperature, the minimum quench energy of this material is clearly greater than that of $\mathrm{Nb}-\mathrm{Ti}$, and recent measurements on cables confirm its stability [6]. Wire can be made multi-filamentary and twisted, and can be made into transposed cables. The material is sensitive to magnetic fields (compared with other types of HTS), but this is not important for a switch, as the wire is not exposed to high fields in the non-inductive winding. Provided barriers are used to encase the $\mathrm{MgB}_{2}$, it is possible to use a variety of materials for the matrix, including the $\mathrm{Cu}-\mathrm{Ni}$ chosen for the $\mathrm{Nb}-\mathrm{Ti}$ conductor considered above. Moreover, $\mathrm{MgB}_{2}$ is comparable in cost to $\mathrm{Nb}$ - Ti and available in long piece lengths. It is therefore worthwhile to estimate $f_{2}$ for a switch using this material.

It is supposed that the switch would be required to operate stably at $4.5 \mathrm{~K}$, so we can put $J_{\text {eng }}=10^{9} \mathrm{~A} / \mathrm{m}^{2}$ [6]. Assuming average $\nu=8 \times 10^{3} \mathrm{~kg} / \mathrm{m}^{3}, \rho=50 \times 10^{8} \Omega \cdot \mathrm{m}$ (stainless steel and copper-nickel matrix), $C_{T}=40 \times 10^{3} \mathrm{~J} / \mathrm{kg}$ (for operation up to $250 \mathrm{~K}$ ), and $\sigma=1.5$, we have $f_{2}=0.95 \times 10^{-6}$ units, to be compared with $0.86 \times 10^{-6}$ units for the $\mathrm{Nb}$-Ti case.

\section{B. Cuprates}

1) Bi-2223 in a Silver-Gold Matrix: This is currently the material of choice for HTS current leads. Its critical temperature is about $105 \mathrm{~K}$, and it is produced in the form of tape, $4 \mathrm{~mm}$ wide, that safely carries about $100 \mathrm{~A}$ at $77 \mathrm{~K}$ in self field. For large currents parallel tapes are required; some transposition could be achieved by winding tapes helically on a cylinder. For an operating temperature of $4.5 \mathrm{~K}$, chosen to enable a fair comparison with $\mathrm{Nb}$-Ti, the $J_{\text {eng }}$ can be taken to be $5 \times 10^{8} \mathrm{~A} / \mathrm{m}^{2}$ (assuming a favorably aligned field of $3 \mathrm{~T}$ or less) [7]. Applying values of $\nu, \rho$ and $C_{T}$ that are dominated by the large $\mathrm{Ag}-5 \mathrm{Au}$ fraction, and $\sigma=1.5$, gives $f_{2}=6.5 \times 10^{-6}$ units. This is much larger than for $\mathrm{Nb}-\mathrm{Ti}$ and $\mathrm{MgB}_{2}$. The material is also expensive, and could only ever be envisaged for small devices.

2) Bi-2212: This material has better current-carrying characteristics than Bi-2223 at $4.5 \mathrm{~K}$ [7]. Other advantages are that it can be made in the form of multi-filamentary wire that can be cabled, and it is not sensitive to the direction of magnetic field. But it requires a mainly silver matrix, so assuming the same $\mathrm{Ag}-5 \mathrm{Au}$ matrix as for $\mathrm{Bi}-2223$, the factor $f_{2}=4.7$.

3) YBCO: The characteristics of this material are such as to make it a more interesting candidate than the bismuth-based materials for switch conductor. It is also undergoing active development for other major applications, and there is a real potential that its present high cost to performance ratio will diminish with time (though never to that of $\mathrm{MgB}_{2}$ ). It shares a drawback with Bi-2223: it is only produced in the form of tape. But the tape can be much wider (up to $12 \mathrm{~mm}$ ), and by using the Roebel method it is possible to assemble a flat conductor having rudimentary transposition. For comparison with the other materials we assume operation at $4.5 \mathrm{~K}$ in a magnetic field of $3 \mathrm{~T}$ parallel to the broad side. For a typical tape structure having stainless steel substrate and cupronickel in place of stabilizing copper, $J_{\text {eng }}=1.5 \times 10^{9} \mathrm{~A} / \mathrm{m}^{2}[7]$. With average $\rho=10 \times 10^{-8}$ [8], and $\nu$ and $C_{T}$ given by averaging values for tape components, we get $f_{2}=1.5 \times 10^{-6}$ units (with $\sigma=1.5$ ). This is not as good as Nb-Ti and $\mathrm{MgB}_{2}$ but much better than Bi-2223 and $\mathrm{Bi}-2212$. Availability in long lengths is presently a problem, and the material is sensitive to the direction of magnetic field-but it is the best material for use above $25 \mathrm{~K}$.

\section{Switching Off the HTS}

The added stability of HTS switch material compared with $\mathrm{Nb}$-Ti, means that more energy is needed to drive the switch 
normal unless it is being run close to the critical current. For discharge of a circuit running at low temperature and at a fraction of its nominal energy, an YBCO-based switch would benefit from having a matrix for which resistivity increases with temperature, in order to improve latching.

\section{SWITCH DESIGN}

The simplest shape for the winding is circular coils, and for fast action the switch should have low inductance. This can be achieved by making a stack of bifilar-wound pancake coils. For rapid re-cooling after activation, pancakes can be separated with permeable insulation.

\section{SAFETy LEADS AND QUENCH STOPPERS}

Unlike the regular current leads that are optimized for continuous stable operation with a minimum of heat transfer to the cryogenic environment, safety leads only have to conduct for the short time when current is extracted. Such leads are made of a resistive alloy conductor such as stainless steel, the goal being to minimize the heat conducted from room temperature to the cryogenic environment when not passing current, yet having sufficient cross-section to avoid melting during an energy discharge. Being in series with the dump resistor these leads are part of the energy extraction system.

The switch system must include quench stoppers to avoid quenching the busbars connected to the switch. These could be copper blocks with sufficient mass and cooling surface.

\section{ApPlication to Strings of Magnets}

It is interesting to envisage adopting this technology to reduce the time constant for discharging the energy stored in strings of magnets without having to design equipment to withstand large voltages. This can be done by splitting the magnet chain into sectors between each of which the current is diverted from the cryogenic to the ambient environment and installing contact breakers in parallel with dump resistors between the terminals of the current leads [9]. As an alternative, with superconducting switches the circuit would appear as shown in Fig. 3. For example, consider a system divided into octants, series-excited with current $5 \mathrm{kA}$, having a stored energy of $40 \mathrm{MJ}$ per octant, and maximum discharge voltage $1 \mathrm{kV}$ [9]. Superconducting switches could be installed between each octant, each switch being equipped with safety leads connected to an external dump resistor. Each such switch would require just 17 $\mathrm{kg}$ of $\mathrm{Nb}-\mathrm{Ti} / \mathrm{Cu}-\mathrm{Ni}$ conductor.

It could also be envisaged to apply the technique to large solenoids and toroids built from a set of identical modules.

The problem of the control of maximum voltage to ground in the event of asymmetric discharge is solved by introducing a virtual earth at the center of some of the dump resistors [10].

\section{CONCLUSION}

Formulae have been derived to enable designers of magnet systems to estimate the required size of superconducting

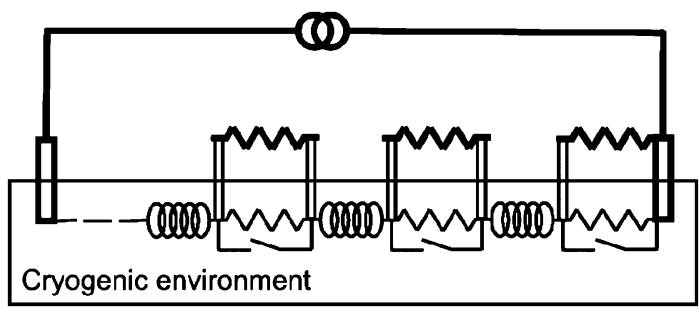

Fig. 3. Schematic of series-connected magnet units with intermediate switches that are opened simultaneously to discharge the stored energy with a short time constant and at reasonable voltage. Symbols are defined in Fig. 1.

switches if they were to be considered for use in energy extraction circuits. The performance of switches based on the use of common HTS materials has been compared with that of classical $\mathrm{Nb}$-Ti switch wire with a cupronickel matrix.

The use of superconducting switches is usually associated with SMES and flux pumps. It is shown that such switches could also find an application handling discharge of magnetic energy stored in large magnet systems, particularly those having series-excited units. A number of practical aspects of such a switch system would nevertheless have to be addressed before committing to its adoption.

\section{ACKNOWLEDGMENT}

The authors thank Karl Hubert Mess for drawing attention to this possibility, and Yifeng Yang and Stephen March for discussions on the use of HTS material for this type of device.

\section{REFERENCES}

[1] H. H. J. ten Kate, H. Pijper, A. Nijhuis, and L. J. M. van de Klundert, "Maximum current and quench sensitivity test of a $40 \mathrm{kA}$ multistrand NbTi/CuNi conductor," in Proc. 9th Int. Conf. on Magnet Technology, Zurich, 1986, pp. 584-587.

[2] S. A. March, "The Application of High Temperature Superconducting Materials to Power Switches," PhD Thesis, Southampton University, , 2009.

[3] R. W. Schmieder, "Superconducting switches using radiation induced quenches," IEEE Trans. Magnetics, vol. MAG-11, pp. 590-593, 1975.

[4] K. Grawatsch, H. Koefler, P. Komarek, H. Kornmann, and A. Ulbricht, "Investigations for the development of superconducting power switches," IEEE Trans. Magnetics, vol. MAG-11, pp. 586-589, 1975.

[5] A. Ballarino, K. H. Mess, and T. Taylor, "Extending the application of HTS in particle accelerators," in EUCAS 2007, J. of Phys.: Conf. Series, 2008, vol. 97, p. 012288, IOP Publishing.

[6] A. Ballarino, "Design of an MgB2 feeder system to connect groups of superconducting magnets to remote power converters," in EUCAS 2009, J. of Phys.: Conf. Series, to be published.

[7] A. Ballarino, "Application of high $T$ c superconductor in accelerators: Status and prospects," J. Cryo. Soc. Japan, vol. 44, no. 9, pp. 411-418, 2009.

[8] H.-I. Du, S.-W. Yim, S.-C. Han, C.-R. Park, and B. S. Han, "Study on the quench protection of YBCO coated conductor," Physica C, vol. 468, pp. 1710-1713, 2008.

[9] K. H. Mess et al., "Quench protection at HERA," in HEACC'92, J. Mod. Phys. A (Proc. Suppl.), 1992, vol. 2A, pp. 304-306.

[10] J.-P. Jensen, "Balance line for HERA proton main ring," in HEACC'92, J. Mod. Phys. A (Proc. Suppl.), 1992, vol. 2A, pp. 318-320. 